\title{
Criminologie
}

\section{De la diffusion de la méthode expérimentale en criminologie}

\section{Pierre Tremblay}

Volume 36, numéro 1, printemps 2003

Police et prévention : évaluation et analyse d'impact

URI : https://id.erudit.org/iderudit/006550ar

DOI : https://doi.org/10.7202/006550ar

Aller au sommaire du numéro

Éditeur(s)

Les Presses de l'Université de Montréal

ISSN

0316-0041 (imprimé)

1492-1367 (numérique)

Découvrir la revue

Citer ce document

Tremblay, P. (2003). De la diffusion de la méthode expérimentale en criminologie. Criminologie, 36(1), 3-7. https://doi.org/10.7202/006550ar d'utilisation que vous pouvez consulter en ligne.

https://apropos.erudit.org/fr/usagers/politique-dutilisation/ 


\title{
De la diffusion de la méthode expérimentale en criminologie
}

\author{
Pierre Tremblay \\ Professeur \\ École de criminologie \\ Université de Montréal \\ pierre.tremblay@umontreal.ca
}

En avril 2002, le Centre international de criminologie comparée et l'École de criminologie de l'Université de Montréal ont organisé le premier Séminaire international de criminologie francophone sur les politiques publiques de sécurité, consacré au thème Prévention du crime, protocoles d'évaluation et analyse d'impact ${ }^{1}$. Un bilan analytique des recherches évaluatives consacrées à la prévention du crime, publié en 1994, notait qu'il était «pratiquement impossible de trouver dans la francophonie d'évaluations scientifiques de programmes de prévention du crime» (C usson et al., 1994). L'objectif du séminaire était de remédier partiellement à cet état de choses. II avait été convenu que les communications qui y seraient présentées seraient soumises, pour fins de publication, à deux revues - la revue C riminologie publiée à M ontréal, d'une part, et la Revue internationale de criminol ogie et de police technique et scientifique publiée à Genève, d'autre part. $D$ ans cette livraison, nous publions une première tranche d'essais et de recherches qui furent présentés lors de ce Séminaire.

1. La réalisation de ce séminaire a été rendue possible grâce à la participation financière de la Stratégie nationale sur la sécurité communautaire et la prévention du crime, du Conseil de recherches en sciences humaines du Canada et du ministère de la Recherche, de la Science et de la Technologie du Q uébec. N ous les en remercions. 
On trouve un nombre croissant d'expérimentalistes en économie, en sociologie et en criminologie. Leurs travaux ont renouvelé les recherches évaluatives des interventions de police. $D$ ans le premier article du présent numéro (À la recherche d'une éval uation "pauvre»), Jean-Paul B rodeur propose un ensemble de neuf tactiques relativement peu coûteuses, susceptibles de constituer une «stratégie d'éval uation pragmatiquement satisfaisante» d'une intervention de police, et qui recoupent largement d'ailleurs le répertoire des consignes évaluatives suggérées par C usson et al. (1994). Les études de cas de M athieu Charest et de Frédéric Lemieux sont exemplaires à cet égard.

L'analyse que consacre $M$ athieu Charest aux effets d'une opération de prévention des cambriolages résidentiels en arrive à quatre résultats importants. II montre tout d'abord qu'une partie des effets induits par les mesures de prévention situationnelle résulte d'une intensification concomitante des efforts répressifs déployés. II indique, ensuite, que dans un protocole évaluatif qui découpe une courbe de criminalité en deux segments (la série temporelle des observations qui précèdent ou qui suivent l'intervention), on surestime (de $50 \%$ en l'occurrence) l'effet d'une opération de prévention lorsqu'on ne prend pas en considération les processus d'autocorrélation inhérents à toute série chronologique. II suggère enfin que, pour remédier à la faiblesse d'un protocole évaluatif qui ne dispose pas d'un site ou d'un groupe contrôle, la meilleure stratégie est de sélectionner une ou plusieurs catégories de délits qui n'ont aucune raison logique ou empirique d'être affectées par l'intervention évaluée (une application concrète du critère d'idonéisme proposé par Jean-Paul Brodeur).

L'étude de Frédéric Lemieux, quant à elle, donne la réplique à celle de $M$ athieu Charest, puisqu'elle montre qu'une partie des effets attribuables à une mobilisation massive des ressources policières en situation de crise ou de désorganisation civile temporaire (la «tempête du verglas» de janvier 1998) est en réalité imputable aux opérations préventives d'indemnisation («chèques de dépannage») de la population touchée directement par les pannes d'électricité. L'étude de Lemieux offre une stratégie ingénieuse pour analyser les effets au jour le jour d'une politique de sécurité publique; elle montre aussi l'intérêt, pour les recherches évaluatives, de procéder à une focalisation temporelle fine («le zoom temporel » de Jean-Paul Brodeur) de leur période d'analyse.

II nous semblait utile d'offrir aux lecteurs du présent numéro un tour d'horizon des recherches évaluatives effectuées en Europe. L'article d'Éric 
H eilmann est consacré aux recherches évaluatives des effets de la vidéosurveillance en Grande-Bretagne. L'article est d'autant plus instructif qu'il expose les principes de base d'une évaluation réaliste formulés par Pawson et Tilley (1997). Ceux-ci s'articulent autour de l'examen de trois éléments fondamentaux : les mécanismes par lesquels une intervention de police peut avoir un effet sur l'évolution de la criminalité, le contexte d'utilisation de cette intervention et les résultats-tendances («outcome pattern ») qui sont produits par ces mécanismes dans des conditions particulières. Les éléments contextuels, dans le cas de la télésurveillance, sont par exemple les conditions d'éclai rage, le nombre et la performance des caméras ou la capacité de réaction du personnel de sécurité ou de police face à la survenance d'un incident. Les mécanismes qui doivent être pris en considération dans une recherche évaluative «sont autant d'hypothèses concernant la façon dont les mesures de prévention peuvent produire des effets». Dans le cas de la télésurveillance, ces mécanismes peuvent être le redéploiement du personnel de sécurité dans les espaces ciblés, la publicité accordée aux arrestations rendues possibles par les enregistrements vidéo ou encore le processus d'adaptation perverse qui amène le personnel de sécurité à relâcher en conséquence leur surveillance physique. Le lecteur lira cet article d'autant plus attentivement qu'une méta-analyse exhaustive et à jour (Welsh et Farrington, 2002) montre, d'une part, que l'impact de la télésurveillance est somme toute très modeste de manière générale (une baisse de $6 \%$ en moyenne des délits signalés) et, d'autre part, très variable selon les contextes d'intervention, le «climat politique» et les catégories de délits.

Les recherches évaluatives ne se limitent pas à une analyse des effets ou à une analyse d'impact. Elles présupposent au départ une conceptual isation préliminaire des indicateurs de performance susceptibles d'être pris en considération pour évaluer la qualité de la prestation des interventions de police ou des corps policiers. B enoît Dupont expose clairement les indicateurs de performance des services de police australiens en matière de sécurité générale (le taux de victimisation, par exemple), de sécurité routière (le taux d'hospital isations causées par les accidents de la route, par exemple), de procédure pénale (la proportion d'affaires où les accusés sont jugés coupables ou le taux de décès en détention provisoire, par exemple) et d'enquêtes criminelles (le taux de non-recouvrement des véhicules volés, par exemple). L'article s'attarde sur les formes que peuvent prendre ces nouveaux instruments d'évaluation, leurs limites et leurs utilités. 0 n trouvera dans la livraison parallèle de la Revue international e de 
criminologie et de police technique et scientifique, entre autres travaux sur lesquels nous ne pouvons, ici, nous attarder, une étude de $M$ arc $O$ uimet et de Paul-Philippe Paré qui propose une stratégie analytique fort ingénieuse pour réhabiliter l'utilisation du «taux de solution » comme indicateur de performance des services policiers.

Law rence Sherman (1992) a fait état de l'éventail des efforts de diplomatie et de la variété des ruses qui avaient été nécessaires pour persuader les corps policiers de consentir à une évaluation expérimentale de trois modalités de règlement des plaintes pour violence conjugale, des enjeux professionnels importants des officiers de police qui avaient accepté de participer à ces expérimentations, des stratégies de marketing de ses recherches et de l'atmosphère houleuse qui régnait dans les chambres d'hôtels enfumées lorsque les résultats de ces expérimentations dans diverses villes américaines se révélèrent contradictoires. De manière analogue, Frédéric 0 cqueteau (C omment éval uer I'impact du travail des policiers de proximité?) reconstitue, pour notre plus grand bénéfice, une «narration suggestive» du labyrinthe des intérêts professionnels, corporatifs et politiques mobilisés par la réforme de police de proximité inaugurée en France en 1997. O n y trouvera aussi des observations instructives sur les effets du déploiement de la police de proximité, sur le volume du contentieux d'outrages et rébellion à l'endroit des forces publiques (imputable en partie à l' «utilisation d'adjoints de sécurité très peu assurés de leur légitimité professionnelle») et sur l'importance cruciale du «climat du système de politique local » comme variable contextuelle susceptible de contribuer ou non à la réussite des efforts déployés par la police de proximité.

D e l'article de Frédéric 0 cqueteau, on retiendra également son exposé de l'étude fascinante que Sylvie Tiévant (2001) a consacré aux savoir-faire policiers. La démarche de Tiévant devrait inspirer une nouvelle génération de recherches éval uatives. Cette démarche, essentiellement inductive, procéderait en quatre étapes: a) identifier une catégorie particulière de prestation de service dont on souhaite évaluer la performance; b) focaliser l'analyse sur les conditions de réussite plutôt que sur les conditions d'échec d'une intervention de police quelconque (principe de base de la recherche évaluative proposé par Ronald V. Clarke, 1993); c) interroger les intéressés eux-mêmes et découvrir les stratégies spécifiques qui expliquent pourquoi certains policiers réussissent mieux que d'autres à accomplir ce travail (principe d'individualisme méthodologique); d) élaboration 
subséquente d'indicateurs mixtes couplant à la fois des indicateurs de résultats et des indicateurs de savoir-faire.

Pour boucler la section thématique de ce numéro, nous offrons en conclusion l'analyse comparative d'Anne Wyvekens (Politiques locales de sécurité françaises, vues d'A mérique...). Cet essai propose une comparaison systématique des finalités distinctes qui guident les réformes de police communautaire en Amérique du $\mathrm{N}$ ord et de police de proximité en France. Anne Wyvekens y développe deux thèses. La première est que la police de proximité constitue une «façon nouvelle de concevoir la prévention », alors que la caractéristique principale de la police communautaire est un «renforcement de la répression». La deuxième thèse est que le principe régulateur de la police de proximité en France est étatiste: « rapprocher les institutions des habitants, tout d'abord pour qu'elles s'occupent d'eux, pour qu'elles les rassurent», d'une part, et transmettre, d'autre part, une pédagogie du droit par le biais d'agents locaux, d'adjoints de sécurité et de médiation sociale (35000 emplois créés depuis 1997).

Le principe régulateur de la police communautaire aux États-U nis, par contre, serait d'établir une «relation forte» entre les services de police et les collectivités locales, où les corps policiers se mettent «en situation de rendre des comptes aux habitants, ce qui leur permettrait, comme en échange, d'obtenir d'eux une contribution - informations, plaintes - à son activité plus spécifiquement répressive». II serait intéressant d'opérationaliser empiriquement ces hypothèses et de procéder à la validation de ce diagnostic.

\section{Références}

Clarke, R.V. (1992). Situational crime prevention. Successful case studies. N ew York: Harrow \& Heston.

Cusson, M ., Tremblay, P., Langelier-Biron, L., 0 uimet, M ., \& Grandmaison, R.

(1994). La prévention du crime. Guide de planification et d'évaluation. Q uébec: $M$ inistère de la Sécurité publique.

Pawson, R., \& Tilley, N. (1997). Real istic evaluation. Londres: Sage.

Sherman, L. (1992). Policing domestic violence: ex periments and dilemmas. N ew York:

Free Press.

Tiévant, S. (2001). Les savoir-faire en police de proximité. Paris: IHESI.

Welsh, B.C., \& Farrington, D.P. (2002). Crime prevention effects of closed circuit television: a systematic review. Londres, H ome O ffice Research Study nº 252. 